\title{
Pleased to Meet You!
}

\author{
Anni-Yasmin Turhan ${ }^{1}$
}

Published online: 25 September 2015

(c) Springer-Verlag Berlin Heidelberg 2015

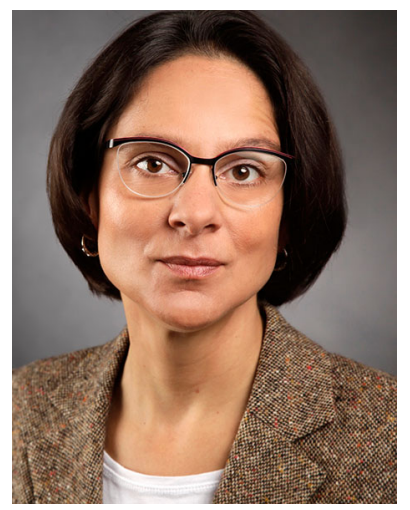

Dear readers,

The field of artificial intelligence is a fast changing field with ideas and concepts from different areas and a rich set of methods. As you know, methods in AI come from areas as diverse such as Psychology, Biology, Engineering and Mathematics-just to name a few. The algorithms and systems developed in our field are applied in areas just as diverse. This journal provides a very good overview of current research in the area of AI with a focus on research from groups situated in German speaking countries. This impression is underlined, for example, by the steadily increasing number of downloads of articles published in the KI Journal. The number of paper downloads has almost doubled in the years from 2011 to 2014.

Earlier this year the editorial board of the KI Journal has undergone quite a bit of a change. Franziska Kügl from Örebro University, Simone Frintrop from Rheinische Friedrich-Wilhelms-Universität Bonn, and Ute Schmid from Otto-Friedrich-Universität Bamberg have left the editorial board after years of great service to the AI community. Now, Britta Wrede from the University of Bielefeld, Sabine Timpf from the University of Augsburg, and

Anni-Yasmin Turhan

anni-yasmin.turhan@tu-dresden.de

1 Faculty of Computer Science, Dresden University of Technology, 01067 Dresden, Germany myself have joined and are still the new kids on the (editor's) block.

Allow me to introduce myself to you. I work in the area of logic-based knowledge representation, where information about an application domain, for instance about Medicine, is modeled by logical formulas. In this field, we investigate reasoning algorithms that use the explicitly given facts to derive new, only implicitly captured ones. Depending on how expressive the underlying logic used for modeling is, the algorithms to deduce the new facts become more "costly" in terms of computational complexity. My work is mainly on formal ontology languages or more precisely, on Description Logics, which are nowadays often included in the wider field of semantic technologies. Here, the trade-off between expressivity of the logic in use and the complexity for reasoning in the respective logic is well-investigated for a wide range of logics and reasoning services.

New application areas typically bring novel requirements and thereby induce the need for new extensions. On the one hand, the formalisms themselves might need to be extended to capture new sorts of information such as temporal or uncertain information. These extensions then necessitate the analysis of the formal properties of the reasoning algorithms devised for these extended logics. On the other hand, the collection of reasoning services offered may need to be extended to meet the new requirements ranging from services for maintenance of knowledge bases to the handling of inconsistent facts.

Often reasoning algorithms are optimized and implemented in reasoner systems which are then employed as components of larger AI applications. Once these systems are "out in the field" and the implemented algorithms are actually being used, novel requirements become evident and raise new research questions. Interestingly, this often 
times happens, if other scientific communities use ontology languages or reasoning systems in an originally unintended way. So, getting to know recent trends and developments is vital to research. This is certainly nothing new to you. However, we tend to develop and discuss new trends mainly in our own scientific communities and mostly on an international level. I'd like to make the point that it is likewise important to disseminate ideas between communities and to be informed about what is going on "next door." To encourage and facilitate such an exchange is a major goal of this journal. To achieve it we need impulses and input from the AI community. In this sense I'd like to resume where the last editorial by Ute Schmid has left us: you need your AI community and your AI community needs you.

At the time of writing, the team around Steffen Hölldobler at Technische Universität Dresden is organising the German AI Conference KI to be held in late September. Together with the KI conference programm chairs, Markus Krötzsch, Riccardo Rosati, and Sebastian Rudolph, we are preparing two weeks that are full of exciting events. This year's edition includes a summer school, a doctoral consortium, a broad collection of workshops and, of course, the KI conference itself-all dedicated to current research in the field of artificial intelligence. I am really looking forward to these events and the discussions taking place in the city of Dresden. As of early September it is not decided yet where the German AI Conference KI 2016 will be held. Let's see where we'll be heading to. I'd be pleased to meet you there!

Sincerely yours,

Anni-Yasmin Turhan

\section{Forthcoming Special Issues}

\subsection{Companion Technologies}

At present, we observe a rapid growth in the development of increasingly complex intelligent systems that serve users throughout all areas of their daily life. They range from classical technical systems such as household devices, cars, or consumer electronics through mobile apps and services to advanced service robots in various fields of application. While many of the rather conventional systems already provide multiple modalities to interact with, the most advanced are even equipped with cognitive abilities such as perception, cognition, and reasoning. However, the use of such complex technical systems and in particular the actual exploitation of their rich functionality remain challenging and quite often lead to users cognitive overload and frustration.
Companion Technologies aim at bridging the gap between the extensive functionality of technical systems and human users individual requirements and needs. They enable the construction of really smartadaptive, flexible, and cooperativetechnical systems by employing a combination of $\mathrm{AI}$ techniques and relying on psychological and neurobiological findings.

The special issue Companion Technologies of the KI Journal aims to present ongoing research, application perspectives, and other insights into an exciting research area emerging across the fields of Artificial Intelligence, Cognitive Psychology, and Cognitive Sciences. Topics of interest include, but are not limited to:

- Computational models of cognitive processes,

- reasoning for adaptive systems,

- user-centered planning,

- multi-modal emotion and motivation recognition,

- knowledge-based humancomputer interaction,

- knowledge-based dialogue management,

- cooperative and adaptive systems.

The KI Journal, published and indexed by Springer, supports a variety of formats including technical articles, project descriptions, surveys, dissertation abstracts, conference reports, and book reviews. Interested authors are asked to contact the guest editors at their earliest convenience:

\section{Prof. Dr. Susanne Biundo-Stephan Daniel Höller \\ Pascal Bercher \\ Institute of Artificial Intelligence \\ Ulm University \\ 89069 Ulm \\ susanne.biundo@uni-ulm.de \\ daniel.hoeller@uni-ulm.de \\ pascal.bercher@uni-ulm.de}

\subsection{Semantic Web}

With more and more data available on the web, the use of semantic technologies is the key to making this knowledge accessible to machines. Thirteen years after the last special issue on the Semantic Web, it is time to review again the advances and state-of-the-art in this area. Several new standards for specifying data and schema information in a machine-processable way have emerged: the basic language to describe data, the resource description framework (RDF) with its schema extension RDFS has been revised in 2014; SPARQL became a widely used query language (SPARQL 1.1 standardised in 2013) and now also supports updates, retrieval of entailed query answers, and federation; the Web Ontology Language OWL has been revised 
and extended to OWL 2; and the rule interchange format RIF (standardized in 2013) allows for expressing rules in a common format. The recent Linked Open Data movement is based on these standards and makes a vast amount of interlinked resources available in the web and for use in semantically enriched applications.

What was still a far-away future when the first Semantic Web special issue appeared became reality: the big search engines now use semantic markup (via RDFa, microdata, or microformats) to improve their search results. With their joint schema.org initiative Google, Microsoft, and Yahoo! provide a unified schema for the structured description of web page content.

Topics of interest include, but are not limited to:

- Management of semantics and data on the Web, including linked data.

- Languages, tools, and methodologies for representing and managing semantics and data on the Web.

- Database, information retrieval, information extraction, natural language processing and artificial intelligence techniques for the Semantic Web.

- Searching and querying the Semantic Web.

- Knowledge representation and reasoning on the Web.

- Cleaning, quality assurance, and provenance of Semantic Web data, services, and processes.

- Semantic Web data analysis.

- Ontology-based data access and integration/exchange on the Web.

- User Interfaces and interaction with semantics and data on the Web.

- Information visualization of Semantic Web data.

- Ontology engineering and ontology patterns for the Web.

- Ontology modularity, mapping, merging, and alignment for the Web.

- Trust, privacy, and security on the Semantic Web.

The KI Journal, published and indexed by Springer, supports a variety of formats including technical articles, project descriptions, surveys, dissertation abstracts, conference reports, and book reviews.

Interested authors are asked to contact the guest editors at their earliest convenience:

\section{Juniorprof. Dr. Birte Glimm \\ Institute of Artificial Intelligence \\ University of Ulm \\ 89069 Ulm \\ birte.glimm@uni-ulm.de}

\section{Prof. Dr. Heiner Stuckenschmidt}

Data- and Web Science Research Group

University of Mannheim

68159 Mannheim heiner@informatik.uni-mannheim.de

\subsection{Landmark-Based Navigation in Cognitive Systems}

The importance of landmarks in human navigation has long been recognized in multiple fields. These include areas involved in the understanding, modelling and supporting wayfinding, spatial knowledge acquisition, and place recognition. From the Psychological, Linguistic and Cognitive Neuroscience viewpoint, the perceived landmarkness of discrete objects vary among individuals. Thus, the key challenge lies in identifying those properties, which remain relevant across a wide range of individual differences, experiences, and behavioural patterns. From the Computer Science, Artificial Intelligence and Cognitive Modelling perspective, formalising these relations in a manner successfully matching the landmark's relevance for humans has proven difficult. Most recently, the increasing volume and accessibility of semantically rich geospatial data has opened new avenues for further progress in this area. The continuing collaboration between these fields is exemplified by the regular conference series on spatial information theory and geospatial science as well as multiple on-going interdisciplinary research projects.

In spite of that, technologies used to support human navigation struggle to incorporate the type of landmark information relevant for the human user. The gap between the human's and the computer's understanding of what constitutes a landmark remains one of the major challenges in the development of spatial systems intuitive in use as well as in modelling navigational behaviour similar to this of a human.

This special issue integrates theoretical, experimental and computational contributions from disciplines involved in the study of landmark-based navigation in cognitive systems. The aim of the issue is to identify new areas for potential interdisciplinary collaboration and we invite applications focusing on, but not limited to, the following topics:

- Automatic, semi-automatic, and crowd-sourced detection of landmarks.

- Modelling of landmark-based navigation.

- Landmark knowledge acquisition and use.

- Communication of landmark-ness.

- Landmark-based approaches for indoor navigation.

- Human-computer interaction with landmark-based systems.

- Ubiquitous computing applications of the landmark concept.

The KI Journal, which is published and indexed by Springer, supports the following lists of formats: Technical 
contributions, research projects, discussions, dissertation abstracts, conference reports, software, and book reviews.

If you are interested in contributing to this special issue, please contact one of the guest editors:
Prof. Dr. Angela Schwering

Dr. Jakub Krukar

Vanessa Joy Anacta

Institute for Geoinformatics

University of Muenster

D-48149 Muenster 